\title{
Transatlantica
}

Revue d'études américaines. American Studies Journal

\section{Le Mausolée de Sally Mann}

De Faces à What Remains : critique et essai sur l'exposition FACES, Galerie Karsten Grève, du 15 novembre 2007 au 19 janvier 2008

\section{Guillemette Minisclou}

\section{Q OpenEdition}

1 Journals

\section{Édition électronique}

URL : https://journals.openedition.org/transatlantica/4311

DOI : $10.4000 /$ transatlantica.4311

ISSN : 1765-2766

Éditeur

Association française d'Etudes Américaines (AFEA)

\section{Référence électronique}

Guillemette Minisclou, «Le Mausolée de Sally Mann », Transatlantica [En ligne], 1 | 2009, mis en ligne le 20 juillet 2009, consulté le 17 septembre 2021. URL : http://journals.openedition.org/transatlantica/ 4311 ; DOI : https://doi.org/10.4000/transatlantica.4311

Ce document a été généré automatiquement le 17 septembre 2021.

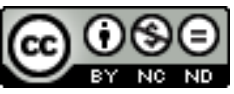

Transatlantica - Revue d'études américaines est mise à disposition selon les termes de la licence Creative Commons Attribution - Pas d'Utilisation Commerciale - Pas de Modification 4.0 International. 


\section{Le Mausolée de Sally Mann}

De Faces à What Remains : critique et essai sur l'exposition FACES, Galerie Karsten Grève, du 15 novembre 2007 au 19 janvier 2008

\section{Guillemette Minisclou}

\section{L'exposition : Faces}

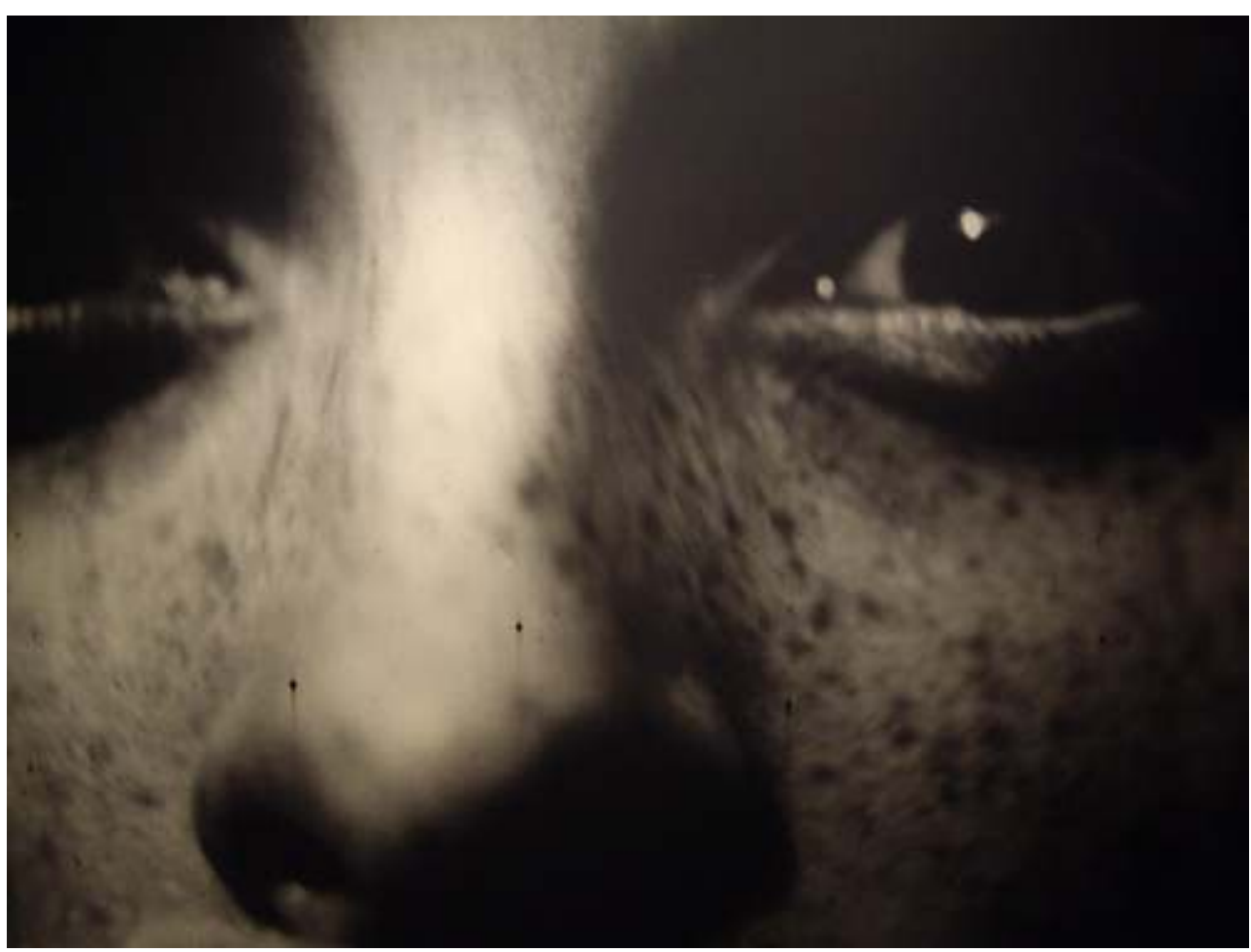

$\mathrm{Au}$ cœur du 3e arrondissement se sont trouvés réunis les 24 portraits des visages des 3 enfants de Sally Mann, Emmet, Jessie et Virginia. L'exposition s'étalait sur 3 niveaux : au rez-de-chaussée étaient disposés les portraits de Virginia, la benjamine ; au niveau 
de l'entresol, Jessie, la cadette et enfin au 1er étage ceux d'Emmet, l'aîné, auxquels s'était ajouté un triptyque des 3 enfants (fig. 1).

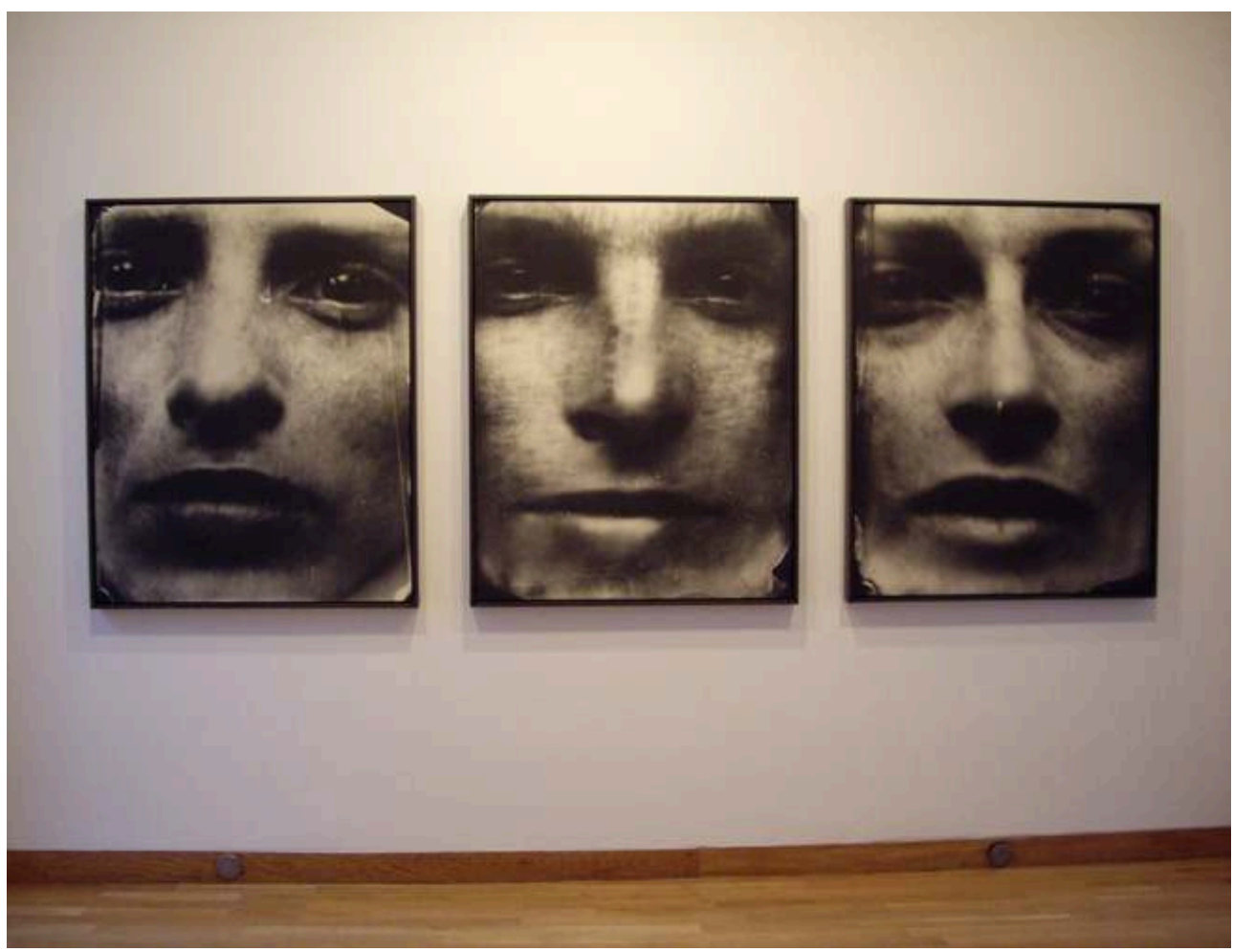

Fig. 1

2 Il est difficile de distinguer le sexe et le visage de chacun tant la technique employée dans ces images peut troubler le spectateur. En effet, certaines parties de l'image tendent à disparaître au profit de la matière photographique. Les photographies sont pour la plupart surexposées, sous-exposées (fig. 4) ou voilées (fig. 2) induisant une disparition partielle ou quasi-totale de l'image et par la même, du sujet photographié. 


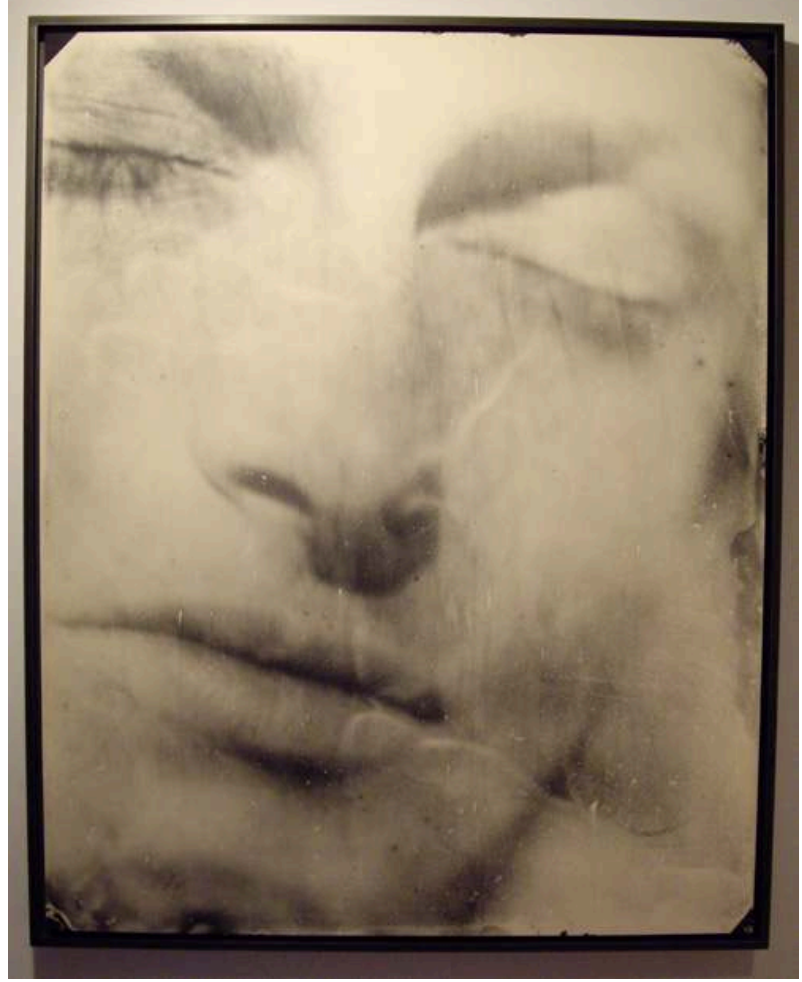

Fig. 4

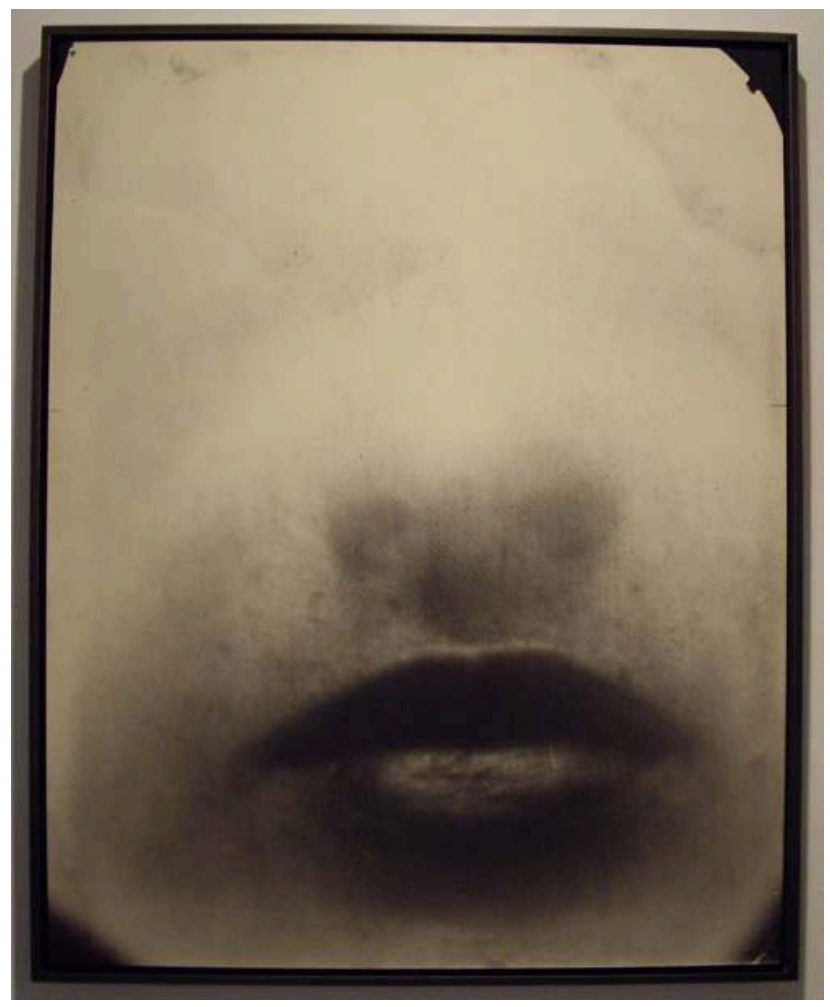

Fig 2

3 Le format imposant des photographies $(127$ x 101, $6 \mathrm{~cm})$ renforce l'impression de disparition de la figure au profit du procédé. A première vue, ce qui frappe irrésistiblement le regard se sont les expressions des visages qui semblent 
fantomatiques, inquiétantes (fig. 3) et morbides (fig. 4). Néanmoins, dès que l'on se rapproche des photographies pour ausculter de plus près leur fabrication, l'œil est alors capté par les nombreuses imperfections et scintillements de la matière qui affectent les tirages.

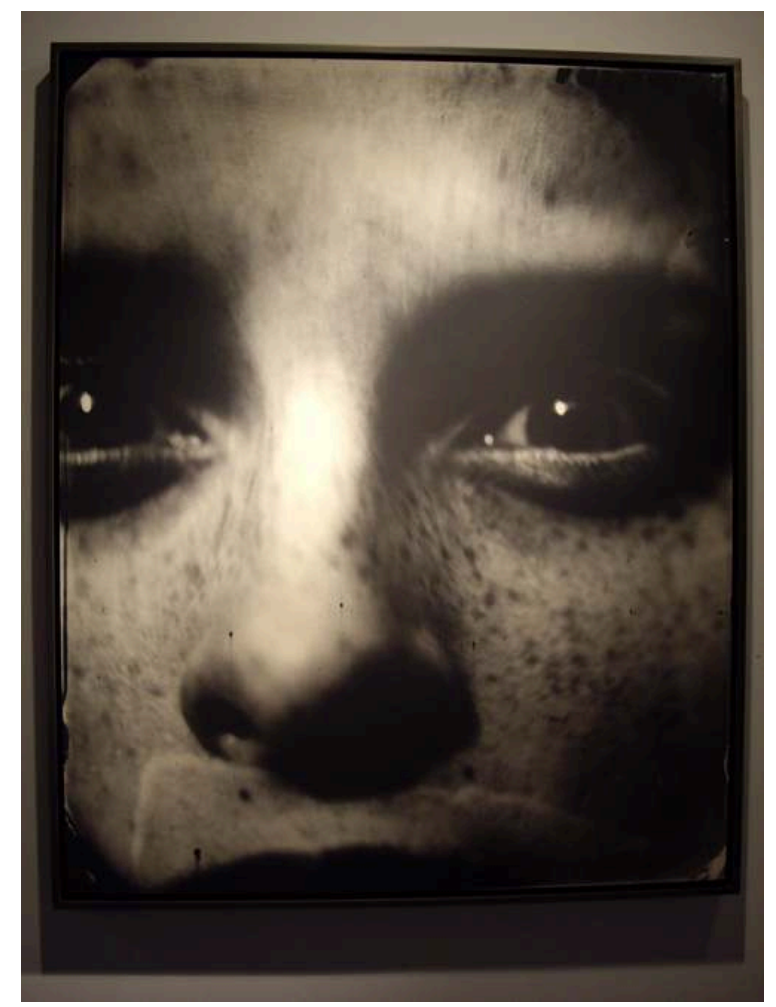

Fig. 3

4 Après Immediate Family, publié chez Aperture en 1992, Sally Mann s'attache de nouveau à dresser le portrait de ses enfants dans un style des plus radical et singulier. Epurés, privés de tout contexte, les portraits paraissent si subordonnés à la technique employée que c'est la matière qui tient lieu de problématique centrale.

5 Rendu visible par le format, la matière photographique est sans équivoque le cœur de cette recherche visuelle où se mêle une façon de faire dix-neuviémiste à une vision contemporaine du portrait.

6 Pour cette exposition, la photographe a uniquement travaillé sur plaque de verre en réalisant les négatifs au collodion humide, procédé ancien découvert par le sculpteur Britannique Frederick Scott Archer (1813-1857) dans les années 1850. Le temps d'exposition est d'approximativement six minutes pour chaque image. Les tons des photographies sont légèrement écrus, leur donnant un aspect ancien, volontairement suranné. Les tirages sont argentiques. Leurs surfaces mats ainsi que le verni « home made » (que la photographe aurait elle-même fabriqué) qui les recouvre forment un voile ajouté à celui, naturel, provoqué par le négatif sur verre.

7 Cet ensemble de données place ce type de photographies à contre-courant d'une donnée actuelle et inévitable, celle de l'ère numérique. « Digital images don't smell » (« les images numériques ne sentent pas ») comme se plait à dire Sally Mann. Et pourtant, cette pichenette verbale - bénigne - de la photographe au monde actuel de la photographie n'est pas réactionnaire en ce sens qu'il permet de réanimer le débat sur le support même des images. La recherche de Sally Mann, affectivement ancrée dans le 
geste photographique et les procédés argentiques en relation à l'Histoire de la photographie, prouve que le support crée à part entière l'image, qu'on ne peut détacher l'image de son procédé et donc que le procédé adhère indubitablement à l'esthétique et l'évocation photographique. En outre, lorsque la photographe recouvre ses tirages argentiques d'un verni, elle participe de par son geste au geste qu'est la photographie argentique et réactive la matière la constituant, matière (ici, le collodion) qu'elle aura au préalable disposé avec habileté sur la plaque de verre

Matière qui s'amuse à jouer avec l'expression même des figures. Ainsi dans l'un des portraits d'Emmet situé au 1er étage (fig. 5), une intense rayure sur le négatif lui fait fermer l'œil droit et de multiples éclaboussures parsèment les alentours de ses lèvres. La matière photographique apparaît comme une cicatrice sur le visage d'Emmet. Une grande partie des images montrent en effet le revêtement instable du négatif sur verre. Les coins des images sont souvent plissés, déchirés (fig. 6) ou cornés ; d'innombrables traces fendent le visage des enfants, dénotant la fragilité de l'image.

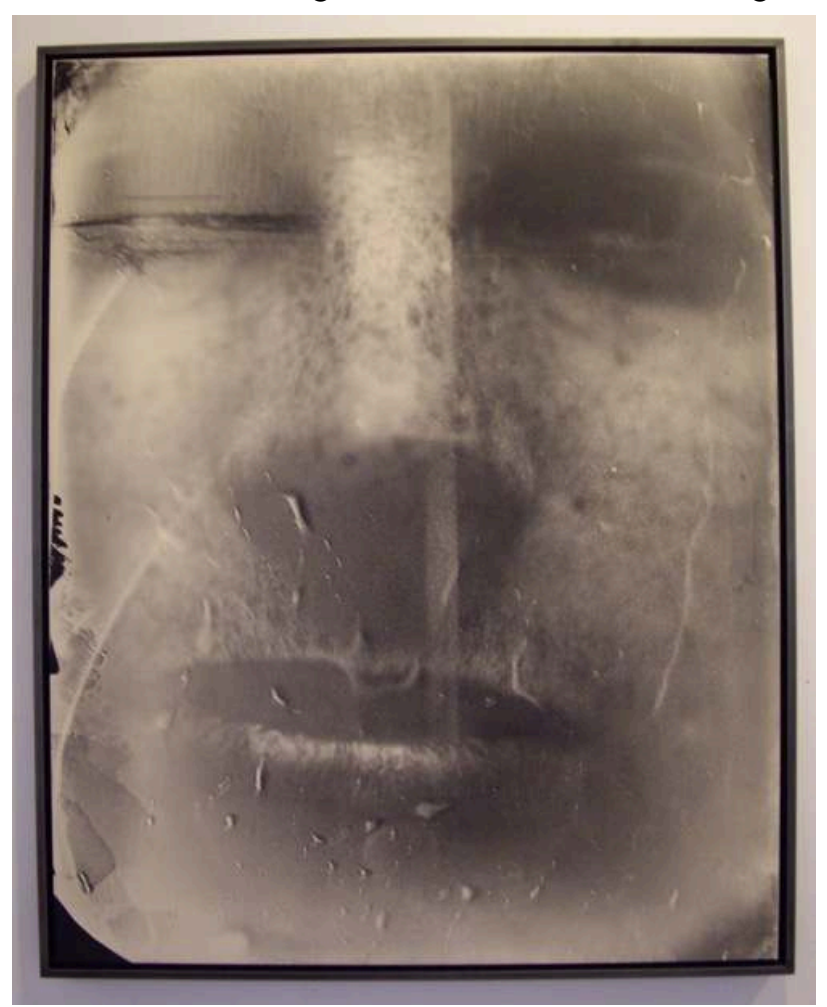

Fig. 5

9 Cette fragilité se reflète aussi intensément dans le regard presque fou (fig. 6 et fig. 3) des figures. Le flou associé au long temps de pose aléatoire amplifie cette évocation à la fragilité, à la démence, au fantomatique, à l'éthéré et au spirite. Les photographies ont sans doute à voir avec le hasard heureux. Ce que Sally Mann nomme le concept de " serendipity » (le don de faire par hasard des découvertes heureuses)

Probablement liées aux propriétés du verre et au souci que l'image ne peut être visible que dans une certaine transparence et inclinaison, l'intime sensation d'un univers friable, imperceptible et spectral se dégage de cette exposition. 


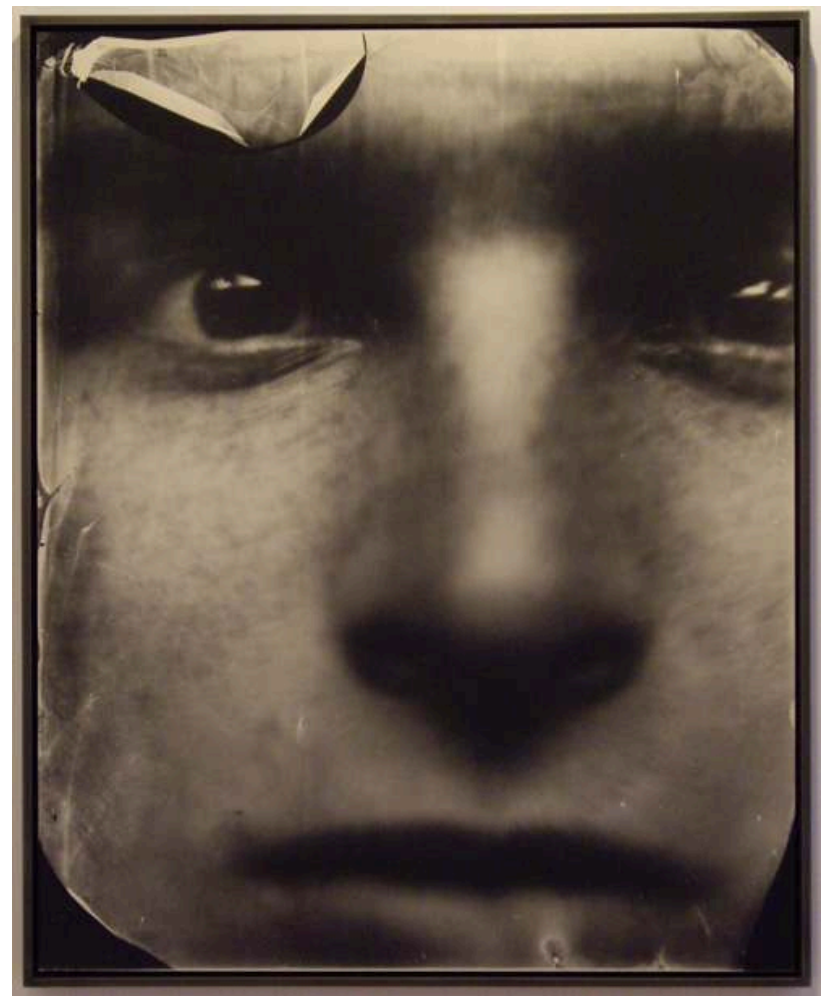

Fig. 6

11 Le spectateur est invité à assister à la disparition des sujets représentés ou, comme s'intitule le livre dans lequel sont présentées ces photographies, à contempler « ce qui reste $"$.

\section{Le livre: What Remains}

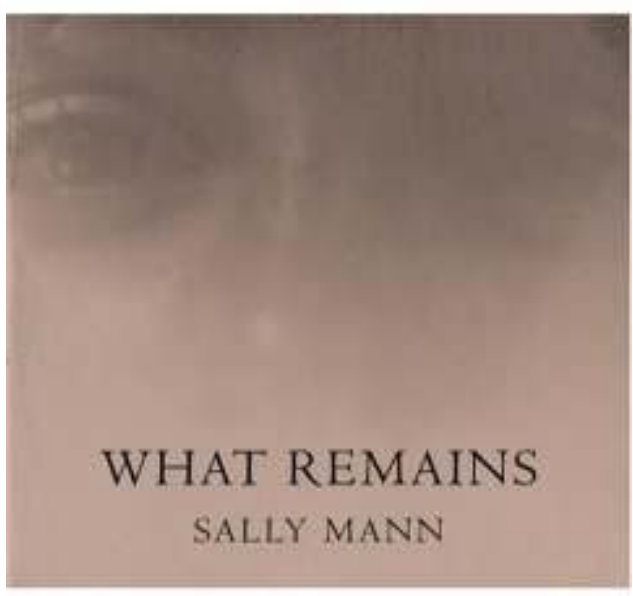

12 L'ensemble des portraits de l'exposition Faces s'intègre à la fin du livre What Remains, publié en 2003 chez Bulfinch Press. La photographe y présente un ensemble de quatre séries d'images, réalisées exclusivement à l'aide du procédé évoqué pour les portraits, comme questionnements (et réponses ?) sur la Nature de la mort. 
13 Les deux premières séries s'intéressent à la mort en tant que processus physique et sont chapitrées par le titre « Matter lent ».

14 Sally Mann est partie des restes (en anglais " remains ») d'une de ses chiennes, Eva. L'animal a été exhumé quelques années après sa mise en terre afin que la photographe puisse, le temps d'une session de prises de vues, se transformer en légiste visuel des atteintes de la mort faites au corps de l'animal.

15 La peau ainsi que les ossements ont été minutieusement photographiés les uns après les autres pour reconstituer la chienne disparue (fig. 7, 8 et 9).

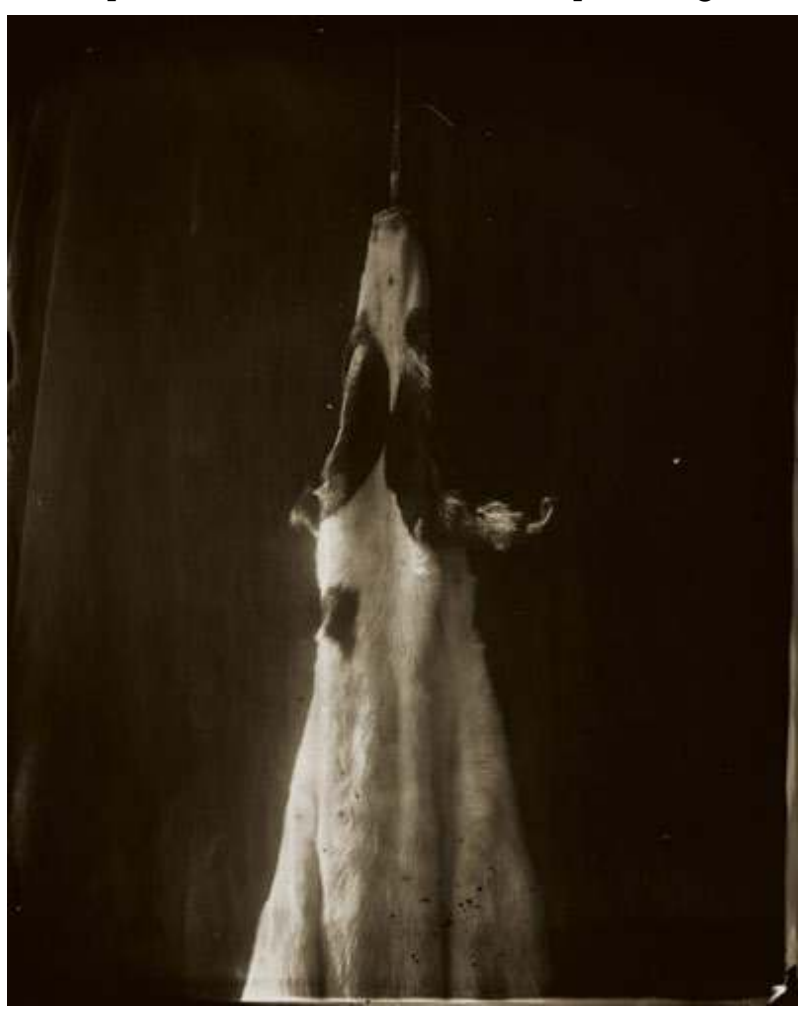

Fig. 7 


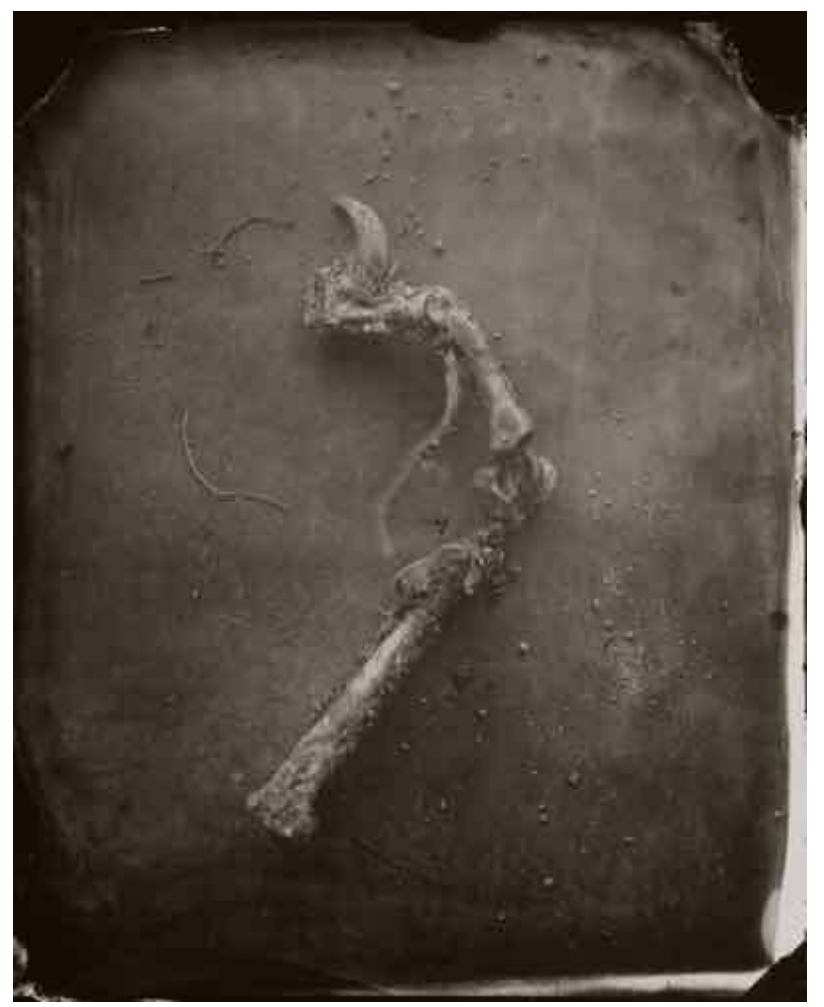

Fig. 8

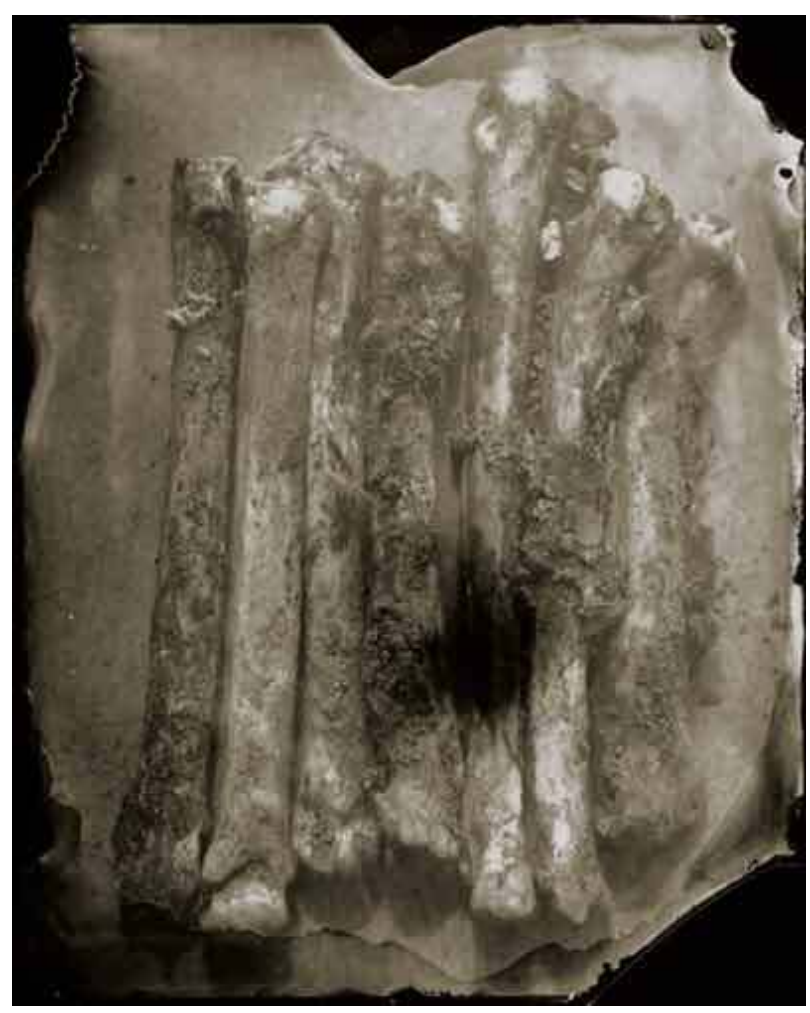

Fig. 9

16 Dans le même attachement, elle s'est penchée sur les corps, humains cette fois, d'une « Body Farm » qui constitue la base de données médico-légale de la police scientifique. Des corps donnés à la science ou tout simplement oubliés sont enterrés ou disposés à 
même le sol afin d'évaluer les différents stades et conditions de la décomposition humaine (fig. 10).

En retournant plusieurs fois sur le site, Sally Mann a pu observer et photographier la progressive désintégration de la chair et l'apparition consécutive de la charpente osseuse, se confondant étrangement, au fur et à mesure du temps, avec la structure organique du sol. Le temps faisant, la terre semble petit à petit absorber les restes humains et former une seule et même unité visuelle (photographies p. 59, 61, 65 et 67).

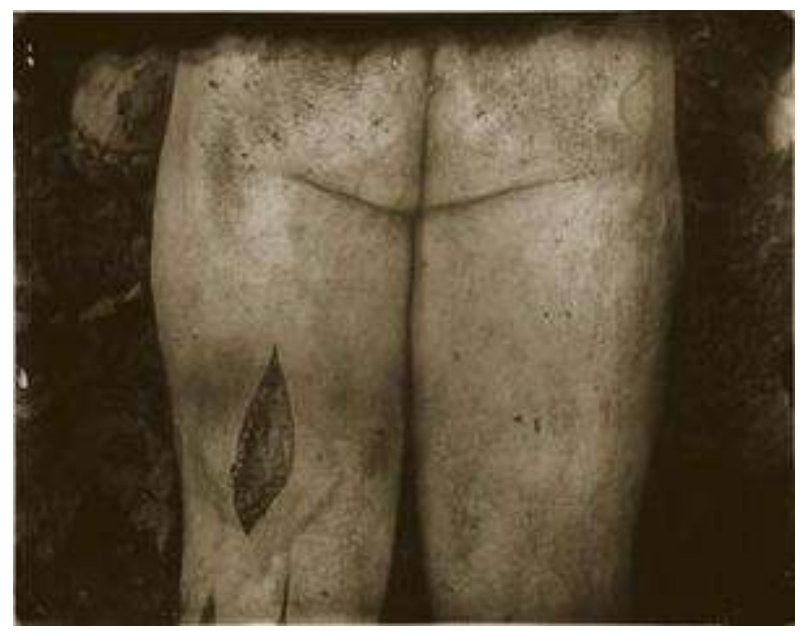

Fig. 10

Cette série vertigineuse sur les corps de la Body Farm montre combien Sally Mann est une photographe obstinée et audacieuse. Passer d'une photographie intimiste avec At Twelve et Immediate Family à un sujet aussi tabou que celui de la décomposition humaine est une preuve, me semble-t-il, du talent hors pair de cette femme photographe.

19 Les deux séries suivantes se focalisent sur la mort comme processus mental, à savoir que la mort ne peut être ressentie/ évaluée que par les vivants et donc qu'elle est intimement liée à la notion d'une mémoire « vivante ».

20 Sally Mann a alors porté son intérêt sur les lieux de la guerre de Sécession Américaine et en particulier sur la bataille d'Antietam, photographiée 140 ans auparavant par l'un des pionniers de la photographie de reportage, Alexander Gardner.

21 La série éponyme regroupe un ensemble d'images qui semblent a priori être purement factuelles, puisqu'elles ne traitent que du lieu même de la bataille, mais qui s'avèrent, de par la noirceur des tirages probablement sous-exposés, prendre place dans l'abstraction pure. On distingue la forme d'un champ, d'un arbre, d'un chemin, d'un creux (fig. 11). 


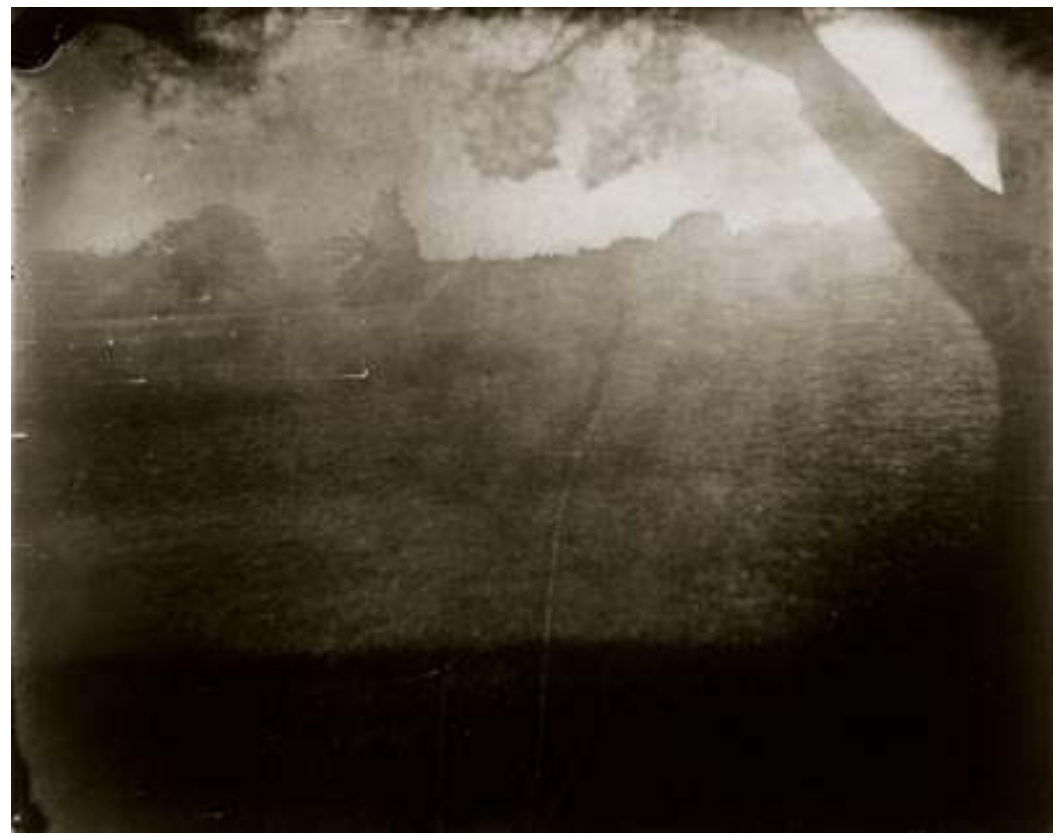

Fig. 11

22 Le paysage est réduit à quelques rares éléments purement formels jusqu'à l'ultime image de la série (photographie p. 99) qui se décompose en deux parties de tonalités grises distinctes que l'on assimile naturellement aux éléments fondateurs qu'est le ciel et la terre.

Que reste-t-il de l'endroit où sont tombés les hommes ? A l'instar de la mémoire qui perdrait de sa force, les images deviennent aveugles.

Le titre "What Remains » revêt deux traductions possibles. C'est à la fois " ce qui reste " au sens strict, c'est à dire " ce qui gît " puisque le terme " remains " peut autant correspondre aux restes (organiques : en l'occurrence aux dépouilles) qu'aux ruines (architecturales : en l'occurrence les vestiges). Néanmoins, l'expression « what remains » possède aussi une valeur symbolique et figurée ; il s'agit de ce qui reste symboliquement (de quelque chose, d'une personne), ce qui est posthume à. Par conséquent, le choix du titre évoque judicieusement le lien étroit qui existe entre l'évocation littérale et figurative des photographies.

L'exposition Faces qui regroupe la série des portraits est reprise dans le livre avec pour intitulé: What Remains, c'est-à-dire le titre même du livre. La conclusion que je déduirai de cette nomination est que Faces constitue l'aboutissement de la recherche de la photographe sur la Nature de la mort. En d'autres termes, les photographies de Faces parcourent les interrogations de la photographe et répondent parfaitement à ce qu'elle a trouvé visuellement au travers des séries précédentes.

26 Sally Mann montre que la mort est à la fois un dispositif de décomposition (« Matter Lent ») et de recomposition ("Antietam »), ces deux dispositifs prennent leur forme ultime dans "What Remains » où le sujet photographié (ce qu'il y a de plus cher à ses yeux : ses propres enfants) rencontre la mémoire, avant même l'heure de la mort. La photographe nous renvoie par la même occasion à notre propre mort : Regardons-nous, nous sommes bien en train de mourir. La photographie prend tout à coup, au travers de cette perspective, une valeur anticipative. Elle semble, d'une part, être le moyen qu'a trouvé la photographe pour échafauder un mausolée anthume aux êtres qui lui sont 
chers et d'autre part, lui a permis d'entrapercevoir la possibilité d'un au-delà athée qui tirerait ses origines de l'écriture photographique.

\section{BIBLIOGRAPHIE}

Sally Mann, What Remains, Bulfinch Press, New York, 2003

Sally Mann, Deep South, Bulfinch Press, New York, 2005

Sally Mann, Immediate Family, Aperture, New York, 1992

Sally Mann, At Twelve: Portraits of Young Women, Aperture, New York, 1988

Beaumont Newhall, Photography: Essays and Images, Moma, New York, 1980

INDEX

Thèmes : Trans'Arts

\section{AUTEUR}

\section{GUILLEMETTE MINISCLOU}

École Nationale Supérieure de la Photographie, Arles 